\title{
Depression And Anxiety In Patients With Juvenile Idiopathic Arthritis: Current Insights And Impact On Quality Of Life, A Systematic Review
}

This article was published in the following Dove Press journal: Open Access Rheumatology: Research and Reviews

\author{
Danielle C Fair \\ Martha Rodriguez (iD) ${ }^{2}$ \\ Andrea M Knight ${ }^{3}$ \\ Tamar B Rubinstein (iD) 4 \\ 'Division of Pediatric Rheumatology, \\ Medical College of Wisconsin: Children's \\ Hospital of Wisconsin, Milwaukee, WI, \\ USA; ${ }^{2}$ Division of Pediatric \\ Rheumatology, Indiana University School \\ of Medicine, Indianapolis, IN, USA; \\ ${ }^{3}$ Division of Pediatric Rheumatology, \\ Hospital for Sick Children, Toronto, ON, \\ Canada; ${ }^{4}$ Division of Pediatric \\ Rheumatology, Albert Einstein College of \\ Medicine: Children's Hospital at \\ Montefiore, Bronx, NY, USA
}

\begin{abstract}
Depression and anxiety are prevalent in children with rheumatologic diseases, including juvenile idiopathic arthritis (JIA). However, prevalence rates and the relationship with disease outcomes, including quality of life are conflicting in the early literature. To review the current literature, determine gaps in our knowledge, and identify areas in need of further investigation, we conducted a systematic review of studies examining depression and anxiety symptoms among children with JIA and the impact these symptoms may have on disease outcomes and quality of life. Six electronic databases were searched up until January 2019. Of 799 potential articles, 60 articles were included with the main focus on 28 articles from 2009 to 2019, to concentrate on the most current evidence. We found that JIA patients experience symptoms of depression and anxiety similar to other childhood chronic diseases and at higher rates than in healthy children. Patients who experience these symptoms have worse quality of life, with some evidence pointing to depression and anxiety symptoms having a greater impact on quality of life than other disease features, such as active joint count. Family members of JIA patients experience high rates of anxiety and depression symptoms which may impact their child's mental health and pain symptoms related to JIA. Conflicting reports of associations between depression/anxiety symptoms and disease features/disease outcomes and a paucity of longitudinal studies investigating the impact of treatment on mental health symptoms indicate areas in need of further research to effectively identify patients at greatest risk of depression and anxiety and to better understand how to treat and prevent these symptoms in youth with JIA. Family mental health should also be considered in investigations concerning mental health and disease outcomes of children with JIA.
\end{abstract}

Keywords: pediatric rheumatology, mental health, mood disorder, arthritis, autoimmune disease

\section{Introduction}

Juvenile idiopathic arthritis (JIA), diagnosed in children under 16, affects an estimated 1.7 to 8.4 million children worldwide. ${ }^{1}$ Incidence rates are estimated to be around 1.6-23 per 100,000. ${ }^{2}$ All races, ethnicities, and ages can be affected. However, females tend to be more frequently affected than males. Children with any of the six different classifications of JIA: oligoarticular ( $<5$ joints), polyarticular ( $>4$ joints), systemic, psoriatic, enthesitis-related (ERA), and undifferentiated can experience musculoskeletal pain and disability, fatigue, and symptoms of psychological distress such as depression and anxiety.

Currently, the incidence of mental health disorders is on the rise within the general United States (US) population. The National Survey on Drug Use and
Correspondence: Danielle C Fair MCW Pediatric Rheumatology, Children's Corporate Center, 999 N 92 ${ }^{\text {nd }}$ St., Suite C465, Wauwatosa, WI 53226, USA

Tel + | 4|4-266-2036

Fax + 4|4-266-6695

Email dfair@mcw.edu 
Health (NSDUH) found that from 2011 to 2016 the prevalence of depression in adolescents increased from $8.3 \%$ to $12.9 \%{ }^{3}$ Children who suffer from chronic disease have higher rates of anxiety and depression than the general population. ${ }^{4-6}$ In addition, children with chronic disease have less favorable courses regarding their mental health symptoms over time. ${ }^{7}$

Reviews of the earlier literature on mental health in children with juvenile arthritis indicated that symptoms of depression and anxiety are prevalent in this population and established the inter-connectedness of parental and child mental health in families affected by JIA. ${ }^{8-10}$ However, studies have reported a wide range of rates for both symptoms and diagnoses of mental health disorders and have conflicted regarding the relationship between these symptoms, disease features, and disease outcomes. Many were limited by small sample sizes, with some studies including fewer than 30 patients, ${ }^{11-13}$ few included significant proportions of racial and ethnic minorities, and few were conducted outside the US and Europe. ${ }^{8}$ Many studies examined children with JIA as part of a general cohort of children with other rheumatologic diseases, ${ }^{11,14-18}$ restricting our understanding of the relationship of psychological symptoms to disease-specific outcomes. In the interim, our treatments and even our classifications of chronic juvenile inflammatory arthritis have changed. The past decade of research in pediatric rheumatology has benefited from the development of longitudinal cohorts (institutional, multicenter, and even international) of both children and adults who suffer from diseases such as JIA. ${ }^{19-21}$ These studies have enhanced our understanding of the epidemiology, risk factors, and outcomes associated with rheumatologic diseases. Given the changes in the field and the general rising prevalence of mental health disorders, we aimed to conduct a systematic review of the recent literature of depression and anxiety in JIA to summarize our knowledge to date, determine gaps in our understanding of depression and anxiety in this population, and identify areas in need of further investigation that will improve care of youth with JIA.

\section{Methods}

\section{Eligibility Criteria}

Studies were included in the review if they (1) involved children, adolescents, and/or adults with JIA, (2) focused on depression and/or anxiety symptoms, and (3) were an original, primary source of research available in English.

\section{Data Sources}

The following databases were searched to identify relevant studies for this systematic review: Ovid MEDLINE, PsycINFO, Web of Science Core Collection, Scopus, the Cumulative Index of Nursing and Allied Health Literature (CINAHL), and Cochrane Database of Systematic Reviews and Cochrane Register of Controlled Trials. The search included literature up until January 2019 and was conducted by a medical librarian in February 2019.

\section{Search Strategy And Study Selection}

The published literature was searched using strategies designed by a medical librarian for the concepts of juvenile idiopathic arthritis or juvenile rheumatoid arthritis and depression or anxiety. Search terms related to juvenile rheumatoid arthritis included the MeSH term "arthritis, juvenile" and keywords related to childhood, childhoodonset or juvenile combined with terms for arthritis including arthrosis, rheumatism, and Still's disease. Search terms related to mental health included the MeSH terms "depression", "depressive disorder, major", "anxiety" and "anxiety disorder", and keywords such as depressive, depressed, anxious, or anxiety.

The initial search was done in Ovid MEDLINE, and the search strategy was peer reviewed by another librarian prior to being translated into subsequent databases. A total of 799 unique references were found. A flowchart of the study identification process is provided in Figure 1. Whereas, full search strategies are provided in the supplementary appendix Table S1.

The remaining 799 articles were organized in Rayyan Qatar Computing Research Institute (QCRI), a web-based app for systematic reviews. ${ }^{22}$ All decisions for study inclusions and exclusions were taken by consensus based on how well the study fit the inclusion criteria. Each abstract was screened by two of three authors (D.F., M.R., and T.R.) in a blinded fashion. Discrepancies were resolved by the third author who had not yet screened the article. This led to a total of 158 articles for potential inclusion in the review. Finally, the full texts of all the potentially included articles were independently reviewed by three authors (D.F., M.R., and T.R.) to determine the final selection of articles. All initially included articles were reviewed, but focus was placed on those written from 2009 to 2019 in order to concentrate on the most current evidence. Full-text articles were excluded for the following reasons: (1) a duplicate article missed in the initial screening, (2) an article was found to be unavailable in 
1374 Records identified through database

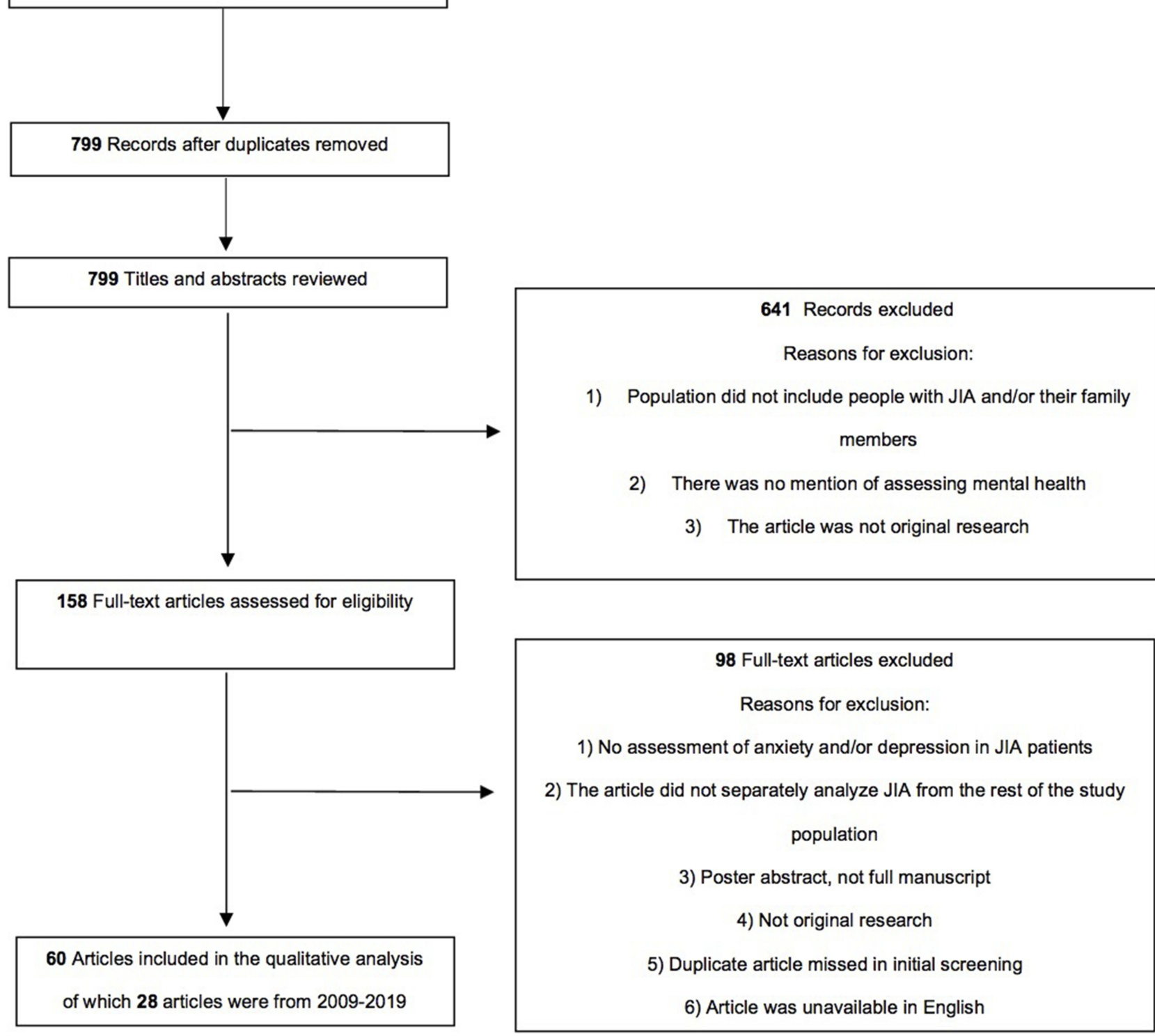

Figure I Flowchart of selection process for study inclusion in the systematic review.

the English language, (3) there was no assessment of anxiety and/or depression in JIA patients, (4) an article did not analyze JIA separately from the rest of the study population, (5) a poster abstract and not a full manuscript, and/or (6) an article was not original research. At the conclusion of the search and screening process, 60 articles were selected for inclusion of which 28 articles were written between 2009 and 2019.

\section{Data Extraction}

The data extracted from the final articles included the title, author(s), year of publication, country in which the study was conducted, patient characteristics (age, gender, sample size, race, ethnicity, disease(s), subtype of JIA, disease duration, disease activity, and medication(s)), family socioeconomic characteristics (parent's educational level and family income), and findings related to depression and anxiety. Reviewers recorded the assessments that were used to screen for anxiety and/or depression and measure the quality of life in both patients and families along with the measures of JIA disease characteristics such as disease activity, mobility, pain, and global health scores. Lastly, reasons for exclusions and limitations were included as well. 


\section{Results}

\section{Study Characteristics}

Overall, 28 articles from 2009 to 2019 were included (Table 1): three-fourths addressed the prevalence of depression, half addressed the prevalence of anxiety, and fewer than one-third addressed the relationship of mental health to JIA disease characteristics or the impact of mental health on patient quality of life. Of the 28 current articles, 24 were conducted in countries outside of the US, including countries outside of North America and Europe (Iran, Turkey, and Egypt).

\section{Population Characteristics}

Twenty-five articles included youth with JIA while 3 articles studied adults. Four studies compared JIA to other chronic childhood diseases. As expected based on the general demographic patterns associated with JIA, study populations included a majority of female patients (range $52-81 \%$ ) with the exception of one study. ${ }^{23}$ Among the included pediatric studies, the mean age was 6.9 to 15.5 years and the age range in the adult studies was 18 to 73 years. Most studies did not report race and/or ethnicities. In the 6 studies that did report race, $\geq 70 \%$ of the subjects were Caucasian or white. ${ }^{24-29}$ JIA disease duration ranged between 3 months to 15 years in children and 10 to 27 years in adults. ${ }^{24-28,30-44}$

JIA subtype was described in 14 studies: 13 included polyarticular, ${ }^{24-26,30,31,35-40,45,46} 11$ included oligoarticular, ${ }^{24,26,30,31,35-40,46} 10$ included systemic, ${ }^{25,26,30,31,35-40}$ and fewer included ERA, psoriatic, undifferentiated, spondyloarthropathy, and other forms of arthritis. ${ }^{25,26,30,31,34-37,40,46}$ JIA subtypes were described by all 3 of the adult studies. ${ }^{42-44}$

\section{Mental Health Measures}

Recent studies mostly relied on patient-reported symptom scales, though parent-reported tools were often used in conjunction (Table 2). Depression was evaluated by a variety of questionnaires, most commonly the Children's Depression Inventory (CDI; in 9/22 depression studies). Other measures used were the Mood and Feelings Questionnaire (MFQ), Beck Depression Inventory-2 (BDI-II), and the Center for Epidemiological Studies Depression Scale for Children (CES-DC). Anxiety was evaluated most commonly by the Screen for Child Anxiety Related Disorders (SCARED; in 3/14 anxiety studies). Other anxiety measures used were the State Trait Anxiety Inventory (STAI), Anxiety Checklist for
Children and Adolescents, and the Self-Administered Psychiatric Scales for Children and Adolescents-Anxiety questionnaire (SAFA-A). To assess symptoms of either depression or anxiety, the Achenbach Child Behavioral Checklist (CBCL), Mini-International Neuropsychiatric Interview for Children (MINI-KID), Schedule for Affective Disorders and Schizophrenia for School-Age Children-Present and Lifetime Version (KSADS-PL), self-report, and psychiatric interviews were used.

\section{Prevalence Of Depressive Symptoms In JIA}

Most studies that used validated screens for depressive symptoms reported prevalences of clinically significant symptoms in the range of $7-36 \%$ in children with JIA. ${ }^{23-26,28,29,35,38,39,41,46,47}$ Reda et al used the MINIKID psychiatric interview and reported that $93 \%$ of the patients had dysthymic disorder while 3\% had major depressive disorder. ${ }^{30}$ El Najjar et al found that all JIA patients scored above the cut-off for clinically significant symptoms on the CES-DC questionnaire. ${ }^{37}$ There were 4 studies that reported no clinically significant depressive symptoms within their study population. $^{27,31-33}$ These studies all used the CDI or BDI, screens that were used to detect prevalence rates of clinically significant symptoms in other studies from $9 \%$ to $36 \%,{ }^{24,25,39}$ but were notable for patient populations from reportedly high socioeconomic levels ${ }^{27,33}$ and/or restricted to either non-acute patients ${ }^{32,33}$ or patients without comorbid psychiatric diagnoses. ${ }^{31}$

Three studies compared JIA patients to healthy controls, of which two found that JIA patients had worse depressive symptoms. ${ }^{23,35}$ Two additional studies reported that youth with JIA had worse anxiety/depressive symptoms than healthy controls; however, these symptoms were not analyzed separately. ${ }^{41,48}$ The exception, Tarakci et al, did not find a significant difference between JIA patients and healthy controls in regards to depression and/or anxiety symptoms; however, they excluded JIA patients with known comorbid psychiatric diagnoses. ${ }^{31}$ Notably, Kayan Ockagoglu et al reported that $28 \%$ of their JIA cohort had a known diagnosis of major depressive disorder. ${ }^{23}$

From the 3 studies that evaluated adults with JIA, two noted a particularly high prevalence of either depression or anxiety symptoms. Barth et al found significantly higher rates of anxiety/depression in JIA adults (18\% in men, $37 \%$ in women) compared to healthy adults, and Rebane 


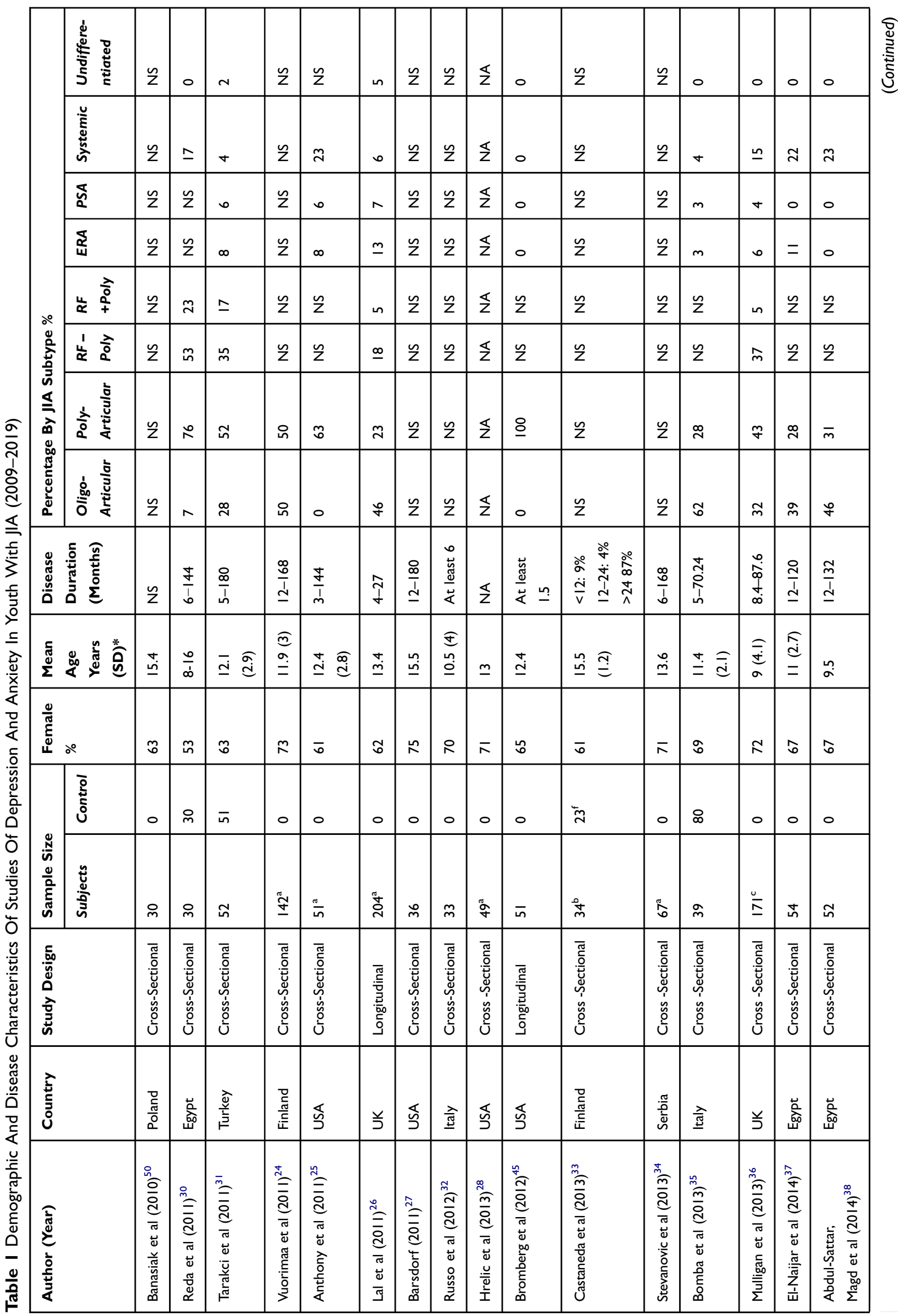




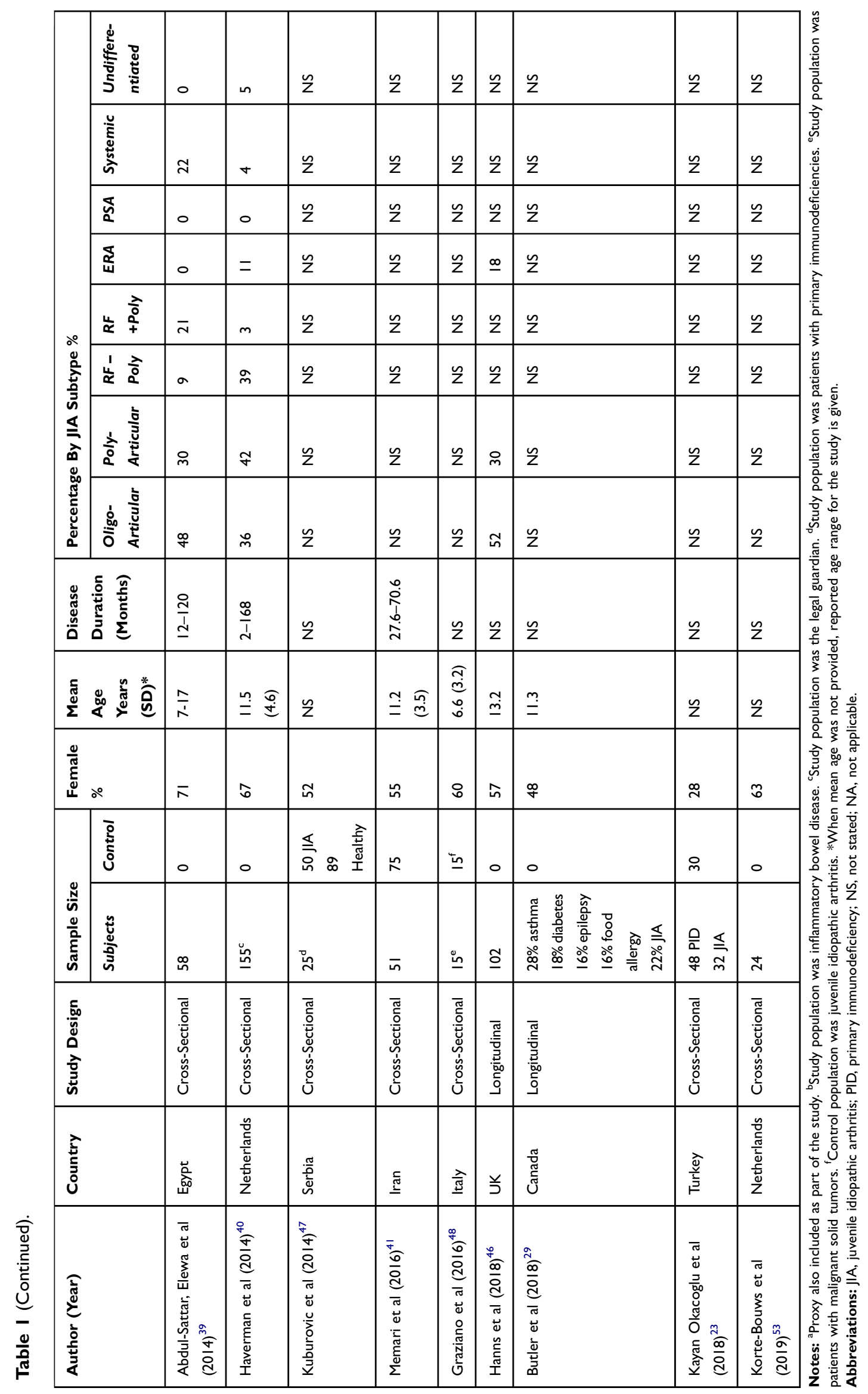




\begin{tabular}{|c|c|c|c|c|c|c|c|c|c|c|c|c|c|c|c|}
\hline 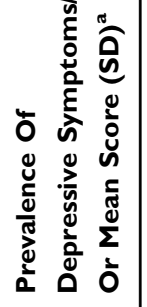 & $\S$ & $\begin{array}{l}\stackrel{\widehat{\infty}}{\stackrel{ \pm}{ \pm}} \\
\bar{\infty}\end{array}$ & $\stackrel{\circ}{\beth}$ & 各 & $\stackrel{\circ}{\stackrel{0}{1}}$ & 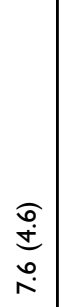 & ஓ゚ & 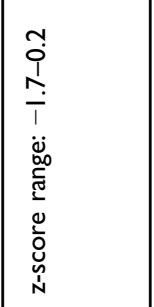 & 芦 & $\stackrel{\circ}{\text { ஸे }}$ & 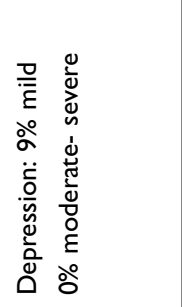 & ○े & $\begin{array}{l}\stackrel{o}{0} \\
\stackrel{\alpha}{\alpha}\end{array}$ & ১্ল & $\frac{\circ}{m}$ \\
\hline 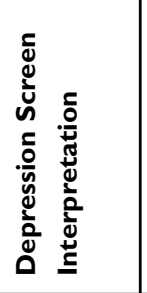 & $\S$ & $\frac{\sigma}{\wedge l}$ & 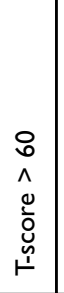 & 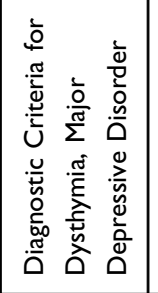 & $\frac{m}{\Lambda}$ & $\frac{\sigma}{\Lambda}$ & $\hat{N}$ & 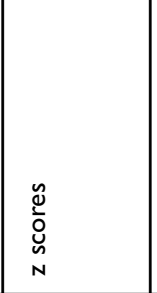 & $\frac{n}{\Lambda 1}$ & $\frac{\sigma}{\Lambda l}$ & 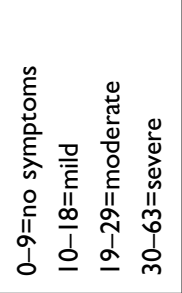 & $\widehat{\lambda}$ & $\frac{n}{N 1}$ & 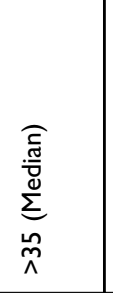 & 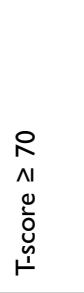 \\
\hline 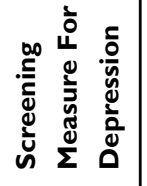 & $\S$ & $\begin{array}{l}\bar{a} \\
\overline{0} \\
0\end{array}$ & $\begin{array}{l}\bar{a} \\
\overline{0}\end{array}$ & $\begin{array}{l}\frac{Q}{\bar{v}} \\
\frac{i}{\bar{z}} \\
\end{array}$ & $\begin{array}{l}\bar{a} \\
\overline{0}\end{array}$ & $\begin{array}{l}\widehat{\widehat{a}} \\
\overline{0}\end{array}$ & 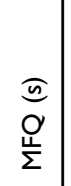 & $\begin{array}{l}\bar{a} \\
\overline{0}\end{array}$ & 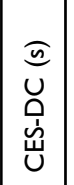 & $\begin{array}{l}\bar{a} \\
\overline{0}\end{array}$ & $\begin{array}{l}\bar{a} \\
\overline{0}\end{array}$ & $\begin{array}{l}\widehat{\Omega} \\
0 \\
\frac{1}{\Sigma}\end{array}$ & 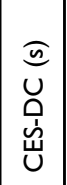 & $\frac{\widehat{a}}{\overline{0}}$ & $\begin{array}{l}\widehat{a} \\
\overline{0}\end{array}$ \\
\hline 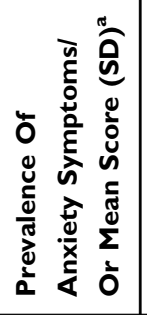 & 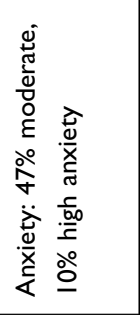 & 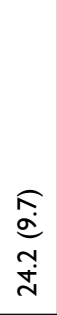 & œ & ஓें & $\grave{\curvearrowright}$ & $\S$ & $\S$ & 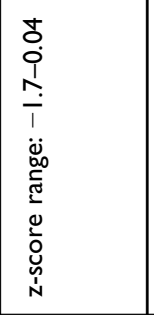 & @ & ○ें & $\S$ & 号 & $\S$ & $\S$ & $\S$ \\
\hline 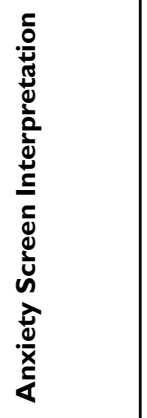 & 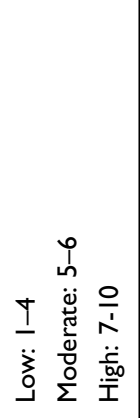 & $\begin{array}{l}\stackrel{\sim}{\sim} \\
\stackrel{N}{*}\end{array}$ & 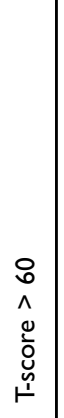 & 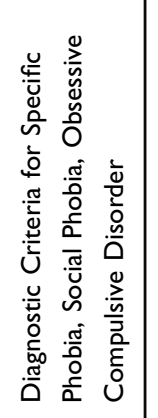 & $\begin{array}{l}\frac{0}{\tilde{J}} \\
\stackrel{!}{!}\end{array}$ & $\S$ & $\S$ & 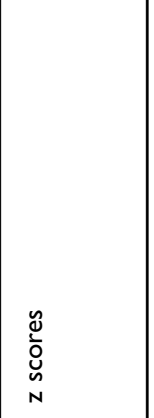 & 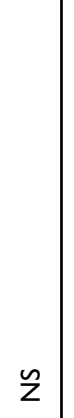 & $\stackrel{m}{N}$ & $\S$ & $\stackrel{\stackrel{\Lambda}{\Lambda}}{\Lambda}$ & $\S$ & $\S$ & $\S$ \\
\hline 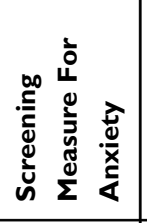 & $\begin{array}{l}\frac{a}{n} \\
\bar{s} \\
\qquad\end{array}$ & 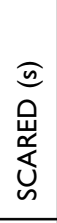 & 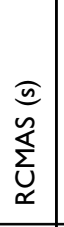 & $\begin{array}{l}\frac{\widehat{n}}{n} \\
0 \\
\frac{\bar{v}}{1} \\
\frac{\vec{z}}{\Sigma}\end{array}$ & $\begin{array}{l}\sqrt{n} \\
\overline{\mathbb{E}} \\
\qquad\end{array}$ & $\S$ & $\S$ & 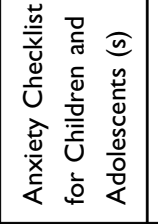 & 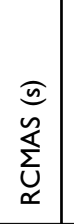 & 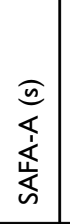 & $\S$ & 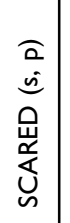 & $\S$ & $\S$ & $\S$ \\
\hline$z \stackrel{\S}{\S}$ & ○ & กี & $\bar{n}$ & ᄋ & $\underline{\tilde{I}}$ & $\stackrel{m}{m}$ & 芩 & $\stackrel{m}{m}$ & 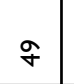 & ळे & $\stackrel{\dddot{N}}{\sim}$ & 요 & है & กี & $\stackrel{\infty}{n}$ \\
\hline 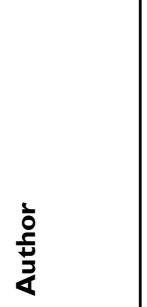 & 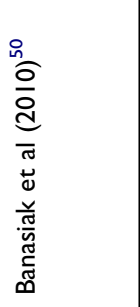 & 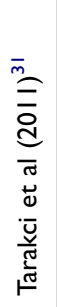 & 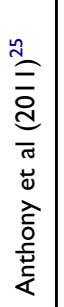 & 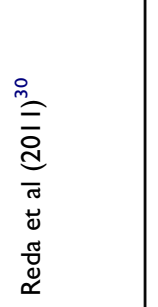 & 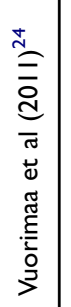 & 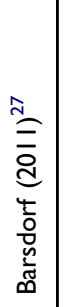 & 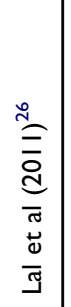 & 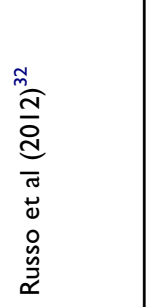 & 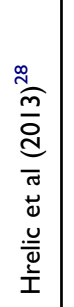 & 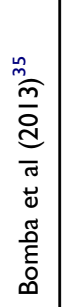 & 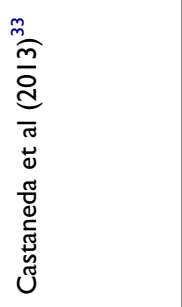 & 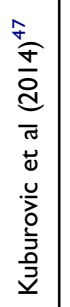 & 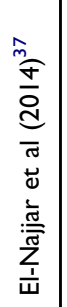 & 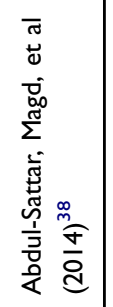 & 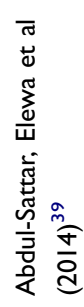 \\
\hline
\end{tabular}




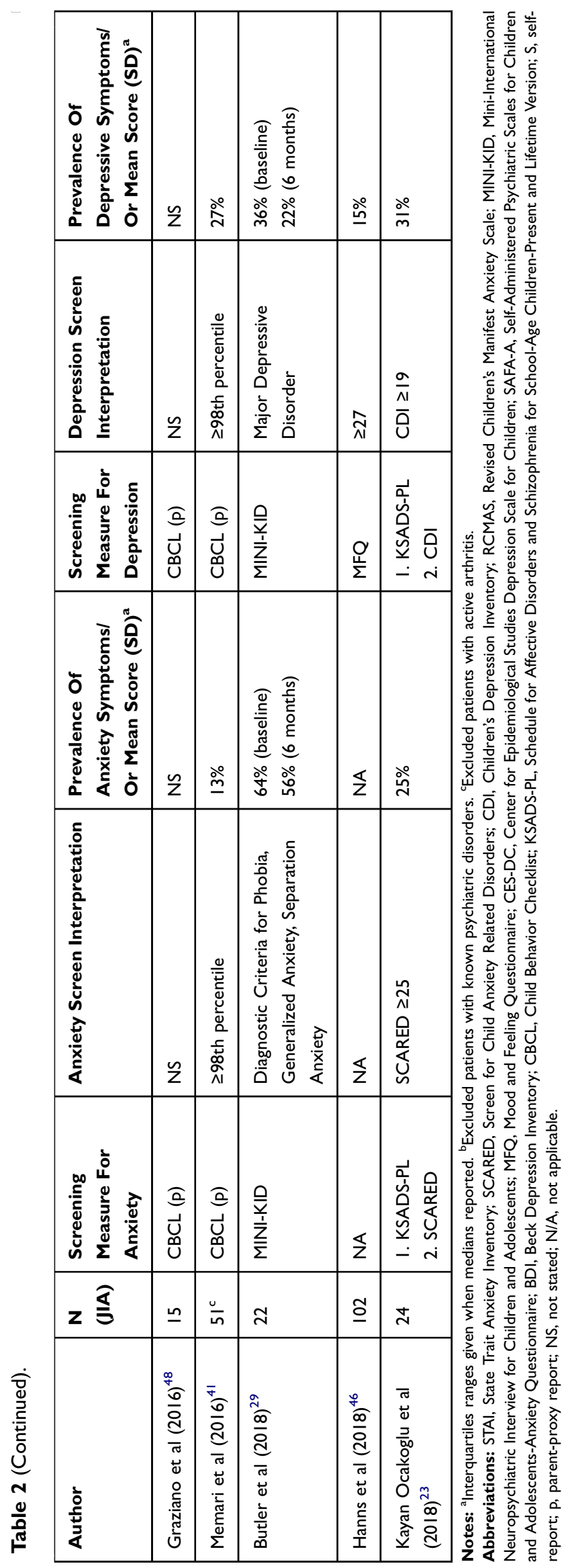

et al reported a depression prevalence of $34 \% .{ }^{43,44}$ In contrast, Raab et al found that only $4.9 \%$ of the adults with JIA self-reported depression, significantly fewer compared to the general population. ${ }^{42}$

While most studies reviewed did not analyze a differential prevalence of depressive symptoms based on age or gender, those that did agree with trends in the general population with older adolescents and females showing worse scores. $^{37,46,49}$

\section{Prevalence Of Anxiety Symptoms In JIA}

The prevalence of clinically significant symptoms of anxiety in youth with JIA ranged from 7\% to $64 \%{ }^{23,25,28,29,35,41,47,50}$ While most studies reported higher rates of depression than anxiety, a few notable studies found the opposite. ${ }^{29,35,47}$ Most studies comparing to healthy controls found higher rates among youth with JIA. ${ }^{35,41,47}$ Kayan Ocakoglu et al found that JIA patients scored worse on the SCARED and were more likely to have diagnoses of anxiety disorders compared to healthy controls, but the difference did not reach statistical significance, likely due to a small sample size. ${ }^{23}$ Tarakci et al, another exception, had a cohort of JIA patients that excluded those with comorbid psychiatric disease. ${ }^{31}$ Bomba et al looked at specific subscales for different types of anxiety (generalized, social, separation, and school anxiety) and found that with each, youth with JIA scored worse than controls. ${ }^{35}$

Butler et al was the only longitudinal study reviewed that examined anxiety and theirs was a pilot study of children with multiple chronic diseases, including a very small number of JIA patients. They found both overall and in JIA patients, high rates of anxiety (up to $36 \%$ ) at baseline and 6-month measurements. ${ }^{29}$

Few studies analyzed the prevalence of anxiety symptoms by age or gender. Russo et al found that children with JIA, ages 6-10 years, scored slightly higher on an anxiety questionnaire than adolescents with JIA, but this finding was not statistically significant. They also noted that psychological unease was reported in all JIA children, ages 6-10 years, compared to $42 \%$ of the adolescents. ${ }^{32}$ Raab et al was the only study to evaluate anxiety in adult patients with JIA and reported that $2 \%$ of the adults with JIA self-reported anxiety disorders in a comorbidity questionnaire. ${ }^{42}$ 


\section{Pathophysiology Of Mental Health In JIA}

In 2011, Capuron et al found an association between chronic low-grade inflammation in elderly individuals with altered neurotransmitter metabolism and disturbed mental well-being. ${ }^{51}$ Then, in 2018 , a review was published discussing the association between inflammation in rheumatoid arthritis and depression. ${ }^{52}$ Therefore, KorteBouws et al decided to evaluate this relationship in JIA by measuring serum samples of a panel of neurotransmitters in children with active JIA. They discovered that ratios between specific neurotransmitters indicated likely elevated activity of enzymes in dopamine and serotonin pathways. This may explain a pathway between active inflammation in JIA and symptoms of depression, anxiety, fatigue, and even cognitive impairment. ${ }^{53}$

Reda et al found that the patterns of cerebral perfusion deficit in patients with JIA are similar to perfusion patterns observed in patients with diagnosed depression and/or anxiety. With single-photon emission computed tomography (SPECT) imaging, they found that children with JIA had a significant decrease in perfusion of the right frontal and right parietal lobes and a significant increase in perfusion of the left parietal and left occipital lobes when compared to healthy controls. These regions of the brain are known to be involved with mood regulation, attention span, and anxiety. ${ }^{30}$

\section{Risk Factors For Depression And Anxiety In Youth With JIA Patient Characteristics}

Hanns et al found that polyarticular patients scored significantly higher than oligoarticular and ERA patients on depressive symptoms. There was no difference in scores for depressive symptoms between the oligoarticular and ERA patients. ${ }^{46}$ Conversely, El-Najjar et al did not find any statistically significant difference between the depressive scores of polyarticular, oligoarticular, systemic, and ERA subtypes. However, all patients scored above the clinically significant cut off for depressive symptoms by the CES-DC. ${ }^{37}$

\section{Disease Activity}

Many of the articles found a correlation between disease activity markers and depression symptoms. Specifically, Hanns et al noted that both a higher active joint count and higher limited joint count correlated with higher depressive scores in the first year, but this relationship was no longer significant after follow up four years later. ${ }^{46}$
Similarly, another study found a statistically significant correlation between depression scores and physician global scores, number of tender joints, number of swollen joints, number of limited joints, and the Juvenile Arthritis Disease Activity Score (JADAS-27). ${ }^{37}$ In Barth et al's study of adult JIA patients, they found that anxiety/ depression symptoms on a health-related quality of life questionnaire were significantly worse in patients with active versus inactive disease. Active disease in this study was also associated with worse mobility, self-care, usual activities, and pain/discomfort. ${ }^{43}$ Conversely, Tarakci et al did not find any significant correlation between depression or anxiety and total active joint count. ${ }^{31}$

Two studies evaluated the relationship between disease duration and anxiety or depression with opposing findings regarding a correlation between duration and symptoms. El-Najjar et al found a strong correlation between worse depression symptoms by CES-DC, ${ }^{37}$ while Tarakci et al found no correlation between disease duration and the CDI. ${ }^{31}$ In part, this may be due to the differing populations of JIA subtypes in these two studies ( $28 \%$ vs $52 \%$ polyarticular, respectively).

\section{Disease Burden}

Three studies found a statistically significant relationship between the Childhood Health Assessment Questionnaire (CHAQ), a patient-reported outcome of physical function, and depression scores. ${ }^{31,37,46}$ Additionally, Hanns et al found that a higher baseline depression score predicted worse disability in patients one and four years later. ${ }^{46}$ On the other hand, Tarakci et al did not find a significant relationship between functional ability level, as measured by physical activities and energy expenditure, and anxiety or depression. ${ }^{31}$

Though most studies supported an association between active disease and worse mental health symptoms, managing disease with medications and blood test monitoring can be a source of psychological burden, as well. Mulligan et al assessed anxiety specific to injections and blood tests in children with JIA on methotrexate for $\geq 6$ months. Over half reported feeling anxious about either. Anxiety about injections was associated with younger age, higher current disease activity, and subcutaneous administration, while anxiety about blood tests was significantly related to a younger age and a shorter duration of time on methotrexate. Importantly, blood test anxiety was an independent predictor for poorer scores in psychosocial quality of life. ${ }^{36}$ Barth 
et al's study of adults found an association between treatment for JIA as an adult and overall lower quality of life. ${ }^{43}$ Notably, treatment for JIA in adulthood may be a marker for progressive, more severe disease.

\section{Pain}

Many studies evaluated the relationship between mental health symptoms and pain and most found an association between pain and either symptoms of anxiety or depression. ${ }^{24,37,46,50}$ The experience of pain may be influenced by particular stress-coping strategies reflective of a child or adolescent's level of anxiety. ${ }^{50}$ Hanns et al noted that a higher baseline depression score predicted worse pain at one and four years later. ${ }^{46}$ Bromberg et al found that daily reported mood was associated with daily measures of pain. ${ }^{45}$ Tarakci et al was the outlier, finding no significant correlation between pain and depression or anxiety. ${ }^{31}$

\section{Impact On Quality Of Life}

Throughout the literature, depression symptoms consistently correlated with impaired overall quality of life. ${ }^{31,34,35,39}$ Abdul-Sattar et al found that over threefourths of patients with significant depression symptoms had poor quality of life. ${ }^{39}$ As expected, studies found that depression symptoms inversely correlated to emotional and social function on measures of quality of life. ${ }^{35}$ Proxy parent reports of symptoms of their children's depression significantly predicted worse quality of life. ${ }^{34}$

Anxiety also appears linked to quality of life in the reviewed literature. Children with JIA with a negative perception of their own life have higher anxiety scores. ${ }^{34}$ However, Stevanovic et al found that after accounting for disease features, pain, and demographics, the association between anxiety and quality of life was lost. ${ }^{35}$

A few studies looked specifically at quality of sleep and school functioning as markers of quality of life. Hrelic et al found that $80 \%$ of the children with JIA reported sleep disturbances of which night waking and parasomnias were significantly correlated with both anxiety and depression symptoms. ${ }^{28}$ Bromberg et al reported that mood moderated the relationship between quality of sleep and pain. ${ }^{45}$ Higher depression scores are strongly associated with poorer school functioning and school absenteeism in youth with JIA. Among JIA patients with significant depression, $88 \%$ had school absences and $69 \%$ had poor school functioning. ${ }^{38}$
A study of adults with JIA evaluated patients on whether they were socially restricted versus non-restricted. The restricted patients were unemployed or on disability while the non-restricted were studying, working, on maternity leave, or in the military. They found that $83 \%$ of the patients were socially non-restricted compared to $16 \%$ restricted. Of the $16 \%$ that were restricted, the majority $(85 \%)$ were unemployed. The socially restricted JIA patients spent less time in leisure non-physical activities, felt more disturbed during leisure time, had lower selfesteem scores, and higher disability scores. They also had statistically significant lower health-related quality of life scores in physical and social functioning as well as emotional well-being compared to the non-restricted. However, they did not find any difference between the depressive scores between the two groups. Of note, 59\% of the restricted patients had polyarticular JIA compared to $47 \%$ of the non-restricted patients; therefore, it could be hypothesized that some differences between the two groups were secondary to worse disease activity in the restricted patients. In contrast, there was no statistically significant difference in disease duration between the two groups. $^{44}$

\section{Impact On Parents And Family}

A few studies addressed the mental health of caregivers of children with JIA. Vuorimaa et al found that $12 \%$ of the caregivers had clinically significant depressive symptoms while $7 \%$ had anxiety. Children's pain was significantly associated with their parents' depressive symptoms and children's depressive and anxiety symptoms moderately correlated with those of their parents. ${ }^{24}$ Russo et al found that $17 \%$ of the mothers of children with JIA and a third of their siblings sought psychological support, while a quarter of mothers endorsed being on an anti-depressant. ${ }^{32}$ Through a health-related quality of life questionnaire, parents of children with active disease scored significantly worse in depressive symptoms than the parents of children with inactive disease. ${ }^{40}$

Haverman et al report that parents of children with JIA perceived their child as more vulnerable compared to parents of healthy children and parents of children with other chronic diseases. ${ }^{40}$ Anthony et al found that higher parent perception of child vulnerability correlated with child depressive symptoms, and children perceived as more vulnerable were also more anxious. In addition, parent daily stress, specifically more intense daily hassles and fewer daily uplifts, predicted higher depressive 
symptoms in their children with JIA. They concluded that children of parents with more daily stress and less stress relief were more susceptible to depressive symptoms, and that parent experiences of daily uplifts or pleasurable events may be protective to the development of depressive symptoms in their children with JIA. ${ }^{25}$ In contrast to the psychopathology described among JIA caregivers in these studies, Graziano et al found that with regards to emotional complaints and psychosocial distress, JIA mothers appeared to have more in common with mothers of healthy controls than with mothers of cancer patients. ${ }^{48}$

\section{Comparison Of Mental Health In JIA And Other Chronic Diseases}

Four articles analyzed mental health in JIA compared to other chronic childhood diseases including inflammatory bowel disease (IBD), primary immunodeficiencies (PIDs), and cancer. $^{23,33,47,48}$ Overall, JIA patients had similar depression/anxiety symptom levels compared to the other chronic diseases, with the exception of IBD patients who were found to be more depressed. ${ }^{33}$ Kayan Ocakoglu et al had children evaluated through a psychiatric interview and found that $63 \%$ of the JIA children, $70 \%$ of the PIDs, and $23 \%$ of the healthy children had some form of a psychiatric disorder (this included disorders other than mood and anxiety such as psychotic, elimination, disruptive, substance abuse, eating disorders, and tic disorders). ${ }^{23}$

\section{Discussion}

Studies of depression and anxiety in youth with JIA conducted in the last 10 years reported that between $7 \%$ and $64 \%$ of the JIA patients have symptoms of anxiety or depression. These prevalence rates are at least as high as the general pediatric population. Most studies found that children with JIA had significantly higher levels of anxiety and/or depressive symptoms than healthy children. ${ }^{23,33,47,48}$ The wide range in symptom prevalence among studies may be attributed to differences in populations, differences in definition or measurement of symptomatology, and differences in methods used to obtain information about patients' anxiety and depression symptoms. In contrast to the earlier literature that depended heavily on parent-reported scales for depression and anxiety, ${ }^{8}$ the reviewed studies mostly relied on patient self-reported symptom scales. Lal et al assessed the level of agreement between adolescents with JIA and their caregivers with regards to mental health and well-being. Adolescents who reported more depressive symptoms were more likely to disagree with their parents about depressive symptoms. ${ }^{26}$ Other reviewed studies also found discrepancies between patient self-reported and parent-reported symptoms of depression/anxiety through screening tools such as the CBCL ${ }^{54,55}$ In pre-adolescent children, there is likely a role for both patient self-report and parent-proxy reports to build a complete picture of a child's mental health.

Likewise, it is still unclear how disease duration may impact the risk of anxiety and depression. Butler et al hypothesized that symptoms would improve after the initial adjustment to illness and found a slight decrease in symptoms at 6 months of follow-up. ${ }^{29}$ This is supported by an earlier study that found that adolescents with lower symptoms of anxiety and depression tended to have longer duration of disease. ${ }^{56}$ El-Najjar et al found the opposite correlation between disease duration and depressive symptoms. ${ }^{37}$ It is possible that longer disease durations are associated with increased damage and changes to function that may increase the risk of developing symptoms of depression. This relationship has been seen with depression in diabetes. ${ }^{57,58}$

Unfortunately, most studies did not perform a separate analysis of mental health symptoms by JIA subtype due to small sample sizes. In one study, polyarticular JIA patients were found to have significantly higher depressive scores than both oligoarticular and ERA patients. ${ }^{46}$ Similarly, an older study reported that adults with RF-negative polyarticular JIA have particularly high rates of depressive symptoms in comparison to adults with other subtypes. ${ }^{59}$ It is possible that polyarticular JIA patients are at an increased risk for depression compared to other subtypes because they commonly suffer from higher active joint counts and higher disease activity which may impair overall function and lead to lower quality of life. On the other hand, ElNajjar et al did not find any significant differences in depressive scores between oligoarticular, polyarticular, systemic, and ERA patients; though in this study, all of the patients scored above the cutoff for significant depressive symptoms. ${ }^{37}$ No included studies examined anxiety symptoms and JIA subtype.

Only a quarter of the studies investigated relationships between other JIA disease characteristics and mental health symptoms. Disease characteristics were assessed in various ways, making comparisons between studies difficult. Most, but not all, of these studies found a positive correlation between higher disease activity and depression/ 
anxiety symptoms. ${ }^{37,43,46}$ Of note, some of the earlier literature conflicts with these findings. ${ }^{55}$

The impact of JIA treatment on symptoms of depression and anxiety was rarely approached in the reviewed studies; however, our search approach was not wide enough to include studies of treatments that reported symptoms of depression and anxiety outside of the main findings highlighted in their abstracts. This is an area deserving of study, especially considering that systemic and brain inflammation have been implicated in psychiatric conditions ${ }^{60}$ such as depression, and anti-tumor necrosis factor (anti-TNF) therapy was recently studied as a potential treatment for refractory depression with mixed results. ${ }^{61}$ In a randomized controlled trial of infliximab for refractory depression, infliximab did not improve depression scores compared to placebo in the overall study population; however, in an exploratory analysis of patients with elevated baseline inflammatory markers, there was a decrease in depression symptoms with infliximab compared to placebo. ${ }^{61}$ In contrast, depression and anxiety have been reported in small numbers in clinical trials of TNF-inhibitors and other treatments used for arthritis in patients with JIA. ${ }^{62-64}$ Hider et al specifically evaluated depression in adults with rheumatoid arthritis on anti-TNF therapy. Although $48 \%$ of the patients had depression at baseline, after a year of anti-TNF therapy the proportion of patients with persistent depression fell to $15 \%$ and only $6 \%$ of the patients on anti-TNF therapy developed newonset depression. Therefore, they concluded that anti-TNF therapy is not likely to adversely affect mood. ${ }^{65}$

Because the vast majority of studies reviewed were cross-sectional, the direction of the relationship between disease activity and mental health symptoms is still poorly described. It is plausible that depressive symptoms may lead to worse disease activity, especially if patients become non-adherent with medical care as a result of their mental health symptoms. In other disease processes, including childhood-onset lupus, medication adherence has been linked to depressive symptoms. ${ }^{66}$ But it is equally plausible that worse disease activity is leading to the development of depressive symptoms. Although most studies were cross-sectional, three studies in adult JIA patients were reviewed to address long-term consequences of JIA. Two were designed to specifically answer the question of how JIA patients fared emotionally as adults and found that $30-40 \%$ of the adults with JIA had symptoms of anxiety or depression. ${ }^{43,44}$ The other study by Raab et al found that adults with JIA had a lower rate of lifetime prevalence of depression compared to a community sample of age-/gender-matched controls. ${ }^{42}$ However, the prior literature also indicates that adults with JIA may have worse anxiety and depression than their healthy peers. ${ }^{59,67,68}$ Therefore, significant psychopathology can impact patients into adulthood and may influence their emotional, social, and overall quality of life. This is similar to what has been reported in children with other chronic diseases such as chronic kidney disease and epilepsy along with many others. ${ }^{4-6,69,70}$ However, in contrast to many other chronic conditions, JIA is characterized by unpredictable flares amidst periods of quiescence and often preceded by significant delays in diagnosis, all of which may further contribute to the development of anxiety and depression.

Although there were two articles that did not find any significant relationship between pain and depression or anxiety symptoms, most of the articles did find that pain was significantly correlated with symptoms. ${ }^{24,31,37,45,46,50}$ This is consistent with earlier literature. ${ }^{18}$ Older literature in JIA reported mixed results with many studies finding a correlation between pain and depression/anxiety symptoms, while some reported weak or no significant association. $^{12,71}$ Hanns et al even found that baseline depressive symptoms predicted worse pain and disability up to 4 years later. ${ }^{46}$ Overall, most of the literature points to an association between pain and psychological symptoms, though much of it does not account for all the potential confounders such as disease severity, activity, disability, and patient demographics.

Physical disability appears to be correlated to mental health symptoms, as well. In two studies, the CHAQ was found to correlate with depressive symptom scores. ${ }^{31,37}$ An earlier cluster analysis of JIA patients by age, anxiety, and depression symptoms found that adolescents with JIA who were more symptomatic of both depression and anxiety also had higher rates of pain, disability, and active joint counts. ${ }^{56}$ Other older literature has reported moderate associations between disability and depression/anxiety symptoms, both in children and adults with JIA. ${ }^{59,72}$ Difficulties in functioning and overall ability to complete activities of daily living may cause children with JIA to feel different than their healthy peers, which may ultimately affect their mood. Additionally, any physical changes or difficulties that are apparent to their peers could lead to bullying which is tied to the development of mental health symptoms. ${ }^{73,74}$ 
The overall quality of life was worse or impaired in JIA patients with higher depressive scores..$^{31,34,35,39}$ Sleep quality of life was also impacted with most JIA patients reporting some sleep disturbance which correlated with anxiety and depressive symptoms, and mood moderated the relationship between poor sleep and pain. ${ }^{28,45}$ This may create a vicious cycle. Pain can interfere with sleep, sleep disturbances can worsen pain, worse pain can lead to feelings of depression, depression can impact sleep and exacerbate pain, and poor sleep can worsen depression further. School quality of life was also impacted by depression, an association that can be explained by how people with depression struggle with participating and performing daily activities. ${ }^{38}$ Prior studies have documented the relationship between depressive symptoms and school impairments in other chronic patient populations such as chronic pain. ${ }^{75}$

Building off the previous literature that indicated that family and parental characteristics and adjustment are associated with child adaptation to illness, ${ }^{76}$ several of the recent studies we reviewed addressed the interconnectivity between family and patient mental health in families affected by JIA. Many of these studies found that parents of children with JIA had clinically significant depressive or anxious symptoms, and that symptoms of depression and anxiety may be worse in family members of children with active disease. ${ }^{24,32,40}$ In addition, more parent daily stress with little stress relief also predicted higher depressive symptoms in their children with JIA. ${ }^{25}$ Vuroimaa et al found that child and parent depressive and anxious symptoms correlated with each other and the child's pain was associated with the parent's depressive symptoms. ${ }^{24}$ This is likely because of shared environmental and genetic predispositions as well as the possibility that one family member's mental health symptoms are influencing the other's. ${ }^{77-79}$ With evidence of significant family psychopathology and psychological distress, it is likely beneficial for the child's mental health to address the parent's mental health, too.

\section{Study Limitations}

Limitations to this review include the challenges of developing a screening process for a broad topic that is discussed with multiple different terms. We may have missed important studies that had keywords related to emotional or mental health and addressed internalized symptoms such as depression and anxiety, without specifically listing them as keywords. Furthermore, we missed articles by limiting our scope to English-language publications.
Knowledge gained by our review was also limited by the limitations of the individual studies. Most of the studies that were included were cross-sectional studies, so symptoms of depression/anxiety were evaluated only at one particular time point. Therefore, trends in symptoms over time could not be evaluated to see if they persisted, worsened, or improved. Consequently, patients who were experiencing symptoms of anxiety/depression outside of the time frame they participated in the study would have been missed. Studies, especially cross-sectional, may introduce non-response bias in which the responses of the people who chose to participate may differ from those who chose to not participate. This may be particularly true for studies involving mental health, where stigma still exists around the topic in some cultures. Lastly, cross-sectional studies allow you to make conclusions about possible associations, but not about causation.

Many of the studies had small sample sizes which can impact the significance and generalizability of the results. Although, most studies were still able to report on statistically significant differences comparing JIA to controls. However, small sample sizes did not provide enough power for most of the studies to complete any analysis about how JIA disease characteristics, medications, or subtypes may be correlated with mental health symptoms. Disease activity was assessed and analyzed with regards to mental health symptoms by varying measures of disease activity making it hard to make comparisons and overall conclusions. Furthermore, some studies only included patients who were in remission or had low/mild disease activity and others excluded patients with known psychiatric diseases. This can lead to potential selection bias, challenging us to compare these and synthesize the disparate observations into a singular understanding of how anxiety and depression impact youth with JIA.

Additionally, few studies reported patient sociodemographics, and US and European countries that reported race/ethnicity of patients still included few racial and ethnic minorities again limiting generalizability. ${ }^{24-29}$

\section{Conclusion}

Recent literature indicates that youth with JIA experience clinically significant symptoms of anxiety and depression similar to youth with other chronic diseases and likely at higher rates than healthy children. These studies have added to the body of literature that address JIA specifically, as opposed to general juvenile rheumatic diseases and help to reinforce the need and importance of mental 
health screening and surveillance specifically in children with JIA. The reviewed studies show an increasing inclusion of work worldwide, even with our limitation in scope to English-language journals. Across diverse geo-cultural backgrounds, it is clear that mental health symptoms are an important part of the experience of JIA and something to which pediatric rheumatology providers must attend.

However, many of the challenges of the early literature still beleaguer the literature of the last ten years. It is likely that large populations of youth with JIA are still not well represented in the literature and this inhibits our understanding of how mental health symptoms impact all patients and which patients are at greatest risk. Disease activity and pain were found in a few articles to correlate with worse depressive and anxiety symptoms; however, the majority of the articles did not perform an analysis of the associations between JIA disease characteristics or subtype with mental health symptoms. More studies are needed to better understand the complex nature of how parents' and other family members' emotional health impacts the affected child's and vice versa. Furthermore, the studies are almost uniformly cross-sectional studies that still leave unanswered questions about how patients progress over time and how the unpredictable and undulating course of JIA disease activity impacts mood and mental health.

As the importance of mental health symptoms to patients and families is continually raised by the arthritis patient community, measurements of these symptoms should be incorporated in the large international longitudinal pediatric rheumatology registries. These studies, which may benefit from a coupling of biospecimen banks, will enhance our understanding of how JIA disease and mental health symptoms interplay over time, how they each impact one another, and may help us understand whether mental health symptoms influence the pathophysiology of inflammatory arthritis or vice versa. This information could provide evidence to target mental health screening efforts, identify novel and effective treatment pathways, and develop individualized therapy plans for youth with JIA.

Though gaps persist in our knowledge of how depression and anxiety influence specific disease factors in JIA, the recent literature clearly points to the role that depression and anxiety symptoms have on quality of life, both for the child with JIA and their family. Pediatric rheumatologists need to consider addressing these symptoms if they wish to promote improved quality of life for their patients.

\section{Acknowledgments}

We would like to acknowledge and thank the medical librarian, Elizabeth Suelzer, for all her hard work in helping us perform the initial literature search and guiding us through the process of a systematic review.

\section{Disclosure}

The authors report no conflicts of interest in this work.

\section{References}

1. Petty RE, Laxer RM, Wedderburn LR. Juvenile Idiopathic Arthritis. In: Petty RE, Laxer RM, Lindsley CB, Wedderburn LR, editors. Textbook of Pediatric Rheumatology. 7th ed. Philadelphia: Elsevier; 2016:188-204.

2. Thierry S, Fautrel B, Lemelle I, Guillemin F. Prevalence and incidence of juvenile idiopathic arthritis: a systematic review. Joint Bone Spine. 2014;81(2):112-117. doi:10.1016/j.jbspin.2013.09.003

3. $\mathrm{Lu}$ W. Adolescent depression: national trends, risk factors, and healthcare disparities. Am J Health Behav. 2019;43(1):181-194. doi:10.5993/AJHB.43.1.15

4. Ferro MA, Boyle MH. The impact of chronic physical illness, maternal depressive symptoms, family functioning, and self-esteem on symptoms of anxiety and depression in children. J Abnormal Child Psychol. 2015;43(1):177-187. doi:10.1007/s10802-014-9893-6

5. Pinquart M, Shen Y. Depressive symptoms in children and adolescents with chronic physical illness: an updated meta-analysis. $J$ Pediatr Psychol. 2011;36(4):375-384. doi:10.1093/jpepsy/jsq104

6. Pinquart M, Shen Y. Anxiety in children and adolescents with chronic physical illnesses: a meta-analysis. Acta Paediatr. 2011;100(8):10691076. doi:10.1111/j.1651-2227.2011.02223.x

7. Ferro MA, Gorter JW, Boyle MH. Trajectories of depressive symptoms during the transition to young adulthood: the role of chronic illness. J Affect Disord. 2015;174:594-601. doi:10.1016/j. jad.2014.12.014

8. LeBovidge JS, Lavigne JV, Donenberg GR, Miller ML. Psychological adjustment of children and adolescents with chronic arthritis: a meta-analytic review. J Pediatr Psychol. 2003;28(1):2939. doi:10.1093/jpepsy/28.1.29

9. Quirk ME, Young MH. The impact of JRA on children, adolescents, and their families. Current research and implications for future studies. Arthritis Care Res. 1990;3(1):36-43.

10. Jaworski TM. Juvenile rheumatoid arthritis: pain-related and psychosocial aspects and their relevance for assessment and treatment. Arthritis Care Res. 1993;6(4):187-196.

11. Schanberg LE, Sandstrom MJ, Starr K, et al. The relationship of daily mood and stressful events to symptoms in juvenile rheumatic disease. Arthritis Care Res. 2000;13(1):33-41.

12. Baildam EM, Holt PJ, Conway SC, Morton MJ. The association between physical function and psychological problems in children with juvenile chronic arthritis. Br J Rheumatol. 1995;34(5):470-477. doi:10.1093/rheumatology/34.5.470

13. Field T, Hernandez-Reif M, Seligman S, et al. Juvenile rheumatoid arthritis: benefits from massage therapy. J Pediatr Psychol. 1997;22 (5):607-617. doi:10.1093/jpepsy/22.5.607

14. Brace MJ, Scott Smith M, McCauley E, Sherry DD. Family reinforcement of illness behavior: a comparison of adolescents with chronic fatigue syndrome, juvenile arthritis, and healthy controls. $J$ Dev Behav Pediatr. 2000;21(5):332-339.

15. Timko C, Baumgartner M, Moos RH, Miller JJ 3rd. Parental risk and resistance factors among children with juvenile rheumatic disease: a four-year predictive study. J Behav Med. 1993;16(6):571-588. 
16. Vandvik IH. Mental health and psychosocial functioning in children with recent onset of rheumatic disease. J Child Psychol Psychiatry. 1990;31(6):961-971. doi:10.1111/j.1469-7610.1990.tb00837.x

17. Daniels D, Moos RH, Billings AG, Miller JJ 3rd. Psychosocial risk and resistance factors among children with chronic illness, healthy siblings, and healthy controls. J Abnorm Child Psychol. 1987;15 (2):295-308. doi:10.1007/bf00916356

18. Kashikar-Zuck S, Goldschneider KR, Powers SW, Vaught MH, Hershey AD. Depression and functional disability in chronic pediatric pain. Clin J Pain. 2001;17(4):341-349.

19. Simard JF, Neovius M, Hagelberg S, Askling J. Juvenile idiopathic arthritis and risk of cancer: a nationwide cohort study. Arthritis Rheum. 2010;62(12):3776-3782. doi:10.1002/art.27741

20. Hinks A, Eyre S, Ke X, et al. Overlap of disease susceptibility loci for rheumatoid arthritis and juvenile idiopathic arthritis. Ann Rheum Dis. 2010;69(6):1049-1053. doi:10.1136/ard.2009.110650

21. Beukelman T, Ringold S, Davis TE, et al. Disease-modifying antirheumatic drug use in the treatment of juvenile idiopathic arthritis: a cross-sectional analysis of the CARRA Registry. J Rheumatol. 2012;39(9):1867-1874. doi:10.3899/jrheum.120110

22. Ouzzani M, Hammady H, Fedorowicz Z, Elmagarmid A. Rayyan-a web and mobile app for systematic reviews. Syst Rev. 2016;5(210). doi:10.1186/s13643-016-0289-2

23. Kayan Ocakoglu B, Karaca NE, Ocakoglu FT, Erermis S. Psychological burden of pediatric primary immunodeficiency. Pediatr Int. 2018;60(10):911-917. doi:10.1111/ped.13675

24. Vuorimaa H, Tamm K, Honkanen V, Komulainen E, Konttinen YT, Santavirta N. Pain in Juvenile Idiopathic Arthritis-a family matter. Childrens Health Care. 2011;40(1):34-52. doi:10.1080/02739615. 2011.537937

25. Anthony KK, Bromberg MH, Gil KM, Schanberg LE. Parental perceptions of child vulnerability and parent stress as predictors of pain and adjustment in children with chronic arthritis. Childrens Health Care. 2011;40(1):53-69. doi:10.1080/02739615.2011.537938

26. Lal SD, McDonagh J, Baildam E, et al. Agreement between proxy and adolescent assessment of disability, pain, and well-being in juvenile idiopathic arthritis. $J$ Pediatri. 2011;158(2):307-312. doi:10.1016/j.jpeds.2010.08.003

27. Barsdorf AI. The relationship of illness representations to caregivers' representations and to self-concept in youths with juvenile arthritis. Diss Abstr Int. 2011;71(12):7699.

28. Hrelic D, Rouster-Stevens K, Gewanter H, Kemper KJ. Sleep problems in JIA patients: Relationships with mood, pain, and functional status and health-related quality of life. J Alt Med Res. 2010;2(1):97106.

29. Butler A, Van Lieshout RJ, Lipman EL, et al. Mental disorder in children with physical conditions: a pilot study. BMJ Open. 2018;8 (1):e019011. doi:10.1136/bmjopen-2017-019011

30. Reda MM, Hosny E, AbuSenna H. Psychiatric morbidity in patients with rheumatoid juvenile arthritis: A SPECT study. Middle East Curr Psychiat. 2011;18(3):132-137. doi:10.1097/01.XME.0000398422.59986.78

31. Tarakci E, Yeldan I, Kaya Mutlu E, Baydogan SN, Kasapcopur O. The relationship between physical activity level, anxiety, depression, and functional ability in children and adolescents with juvenile idiopathic arthritis. Clin Rheumatol. 2011;30(11):1415-1420. doi:10.1007/s10067011-1832-0

32. Russo E, Trevisi E, Zulian F, et al. Psychological profile in children and adolescents with severe course Juvenile Idiopathic Arthritis. Sci World J. 2012;2012:841375. doi:10.1100/2012/841375

33. Castaneda AE, Tuulio-Henriksson A, Aronen ET, Marttunen M, Kolho KL. Cognitive functioning and depressive symptoms in adolescents with inflammatory bowel disease. World $J$ Gastroenterol. 2013;19(10):1611-1617. doi:10.3748/wjg.v19.i10.1611

34. Stevanovic D, Susic G. Health-related quality of life and emotional problems in juvenile idiopathic arthritis. Qual Life Res. 2013;22 (3):607-612. doi:10.1007/s11136-012-0172-0
35. Bomba M, Meini A, Molinaro A, et al. Body experiences, emotional competence, and psychosocial functioning in juvenile idiopathic arthritis. Rheumatol Int. 2013;33(8):2045-2052. doi:10.1007/s00296-013-2685-4

36. Mulligan K, Kassoumeri L, Etheridge A, Moncrieffe H, Wedderburn LR, Newman S. Mothers' reports of the difficulties that their children experience in taking methotrexate for Juvenile Idiopathic Arthritis and how these impact on quality of life. Pediatr Rheumatol Online J. 2013;11(1):23. doi:10.1186/1546-0096-11-23

37. El-Najjar AR, Negm MG, El-Sayed WM. The relationship between depression, disease activity and physical function in juvenile idiopathic arthritis patients in Zagazig University Hospitals - Egypt. Egypt Rheumatologist. 2014;36(3):145-150. doi:10.1016/j.ejr.2014.01.001

38. Abdul-Sattar A, Magd SA, Negm MG. Associates of school impairment in Egyptian patients with juvenile idiopathic arthritis: Sharkia Governorate. Rheumatol Int. 2014;34(1):35-42. doi:10.1007/s00296013-2871-4

39. Abdul-Sattar AB, Elewa EA, El-Shahawy Eel D, Waly EH. Determinants of health-related quality of life impairment in Egyptian children and adolescents with juvenile idiopathic arthritis: Sharkia Governorate. Rheumatol Int. 2014;34(8):1095-1101. doi:10.1007/s00296-014-2950-1

40. Haverman L, van Oers HA, Maurice-Stam H, Kuijpers TW, Grootenhuis MA, van Rossum MA. Health related quality of life and parental perceptions of child vulnerability among parents of a child with juvenile idiopathic arthritis: results from a web-based survey. Pediatr Rheumatol Online J. 2014;12:34. doi:10.1186/15460096-12-34

41. Memari AH, Chamanara E, Ziaee V, Kordi R, Raeeskarami SR. Behavioral problems in Juvenile Idiopathic Arthritis: a controlled study to examine the risk of psychopathology in a chronic pediatric disorder. Int J Chronic Dis Print. 2016;2016:5726236.

42. Raab A, Sengler C, Niewerth M, et al. Comorbidity profiles among adult patients with juvenile idiopathic arthritis: results of a biologic register. Clin Exp Rheumatol. 2013;31(5):796-802.

43. Barth S, Haas JP, Schlichtiger J, et al. Long-term health-related quality of life in german patients with juvenile idiopathic arthritis in comparison to german general population. PLoS One. 2016;11(4): e0153267. doi:10.1371/journal.pone.0153267

44. Rebane K, Ristolainen L, Relas H, et al. Disability and health-related quality of life are associated with restricted social participation in young adults with juvenile idiopathic arthritis. Scand J Rheumatol. 2018;48:1-9.

45. Bromberg MH, Gil KM, Schanberg LE. Daily sleep quality and mood as predictors of pain in children with juvenile polyarticular arthritis. Health Psychol. 2012;31(2):202-209. doi:10.1037/a0025075

46. Hanns L, Cordingley L, Galloway J, et al. Depressive symptoms, pain and disability for adolescent patients with juvenile idiopathic arthritis: results from the Childhood Arthritis Prospective Study. Rheumatology. 2018;57 (8):1381-1389. doi:10.1093/rheumatology/key088

47. Kuburovic NB, Pasic S, Susic G, et al. Health-related quality of life, anxiety, and depressive symptoms in children with primary immunodeficiencies. Patient Prefer Adher. 2014;8:323-330. doi:10.2147/ PPA.S58040

48. Graziano S, Rossi A, Spano B, Petrocchi M, Biondi G, Ammaniti M. Comparison of psychological functioning in children and their mothers living through a life-threatening and non life-threatening chronic disease: A pilot study. J Child Health Care. 2016;20 (2):174-184. doi:10.1177/1367493514563854

49. Mojtabai R, Olfson M, Han B. National trends in the prevalence and treatment of depression in adolescents and young adults. Pediatrics. 2016;138(6):e20161878-e20161878. doi:10.1542/peds.2016-1878

50. Banasiak B, Smolewska E, Zygmunt A, Lipińska J, BiernackaZielińska M, Stańczyk J. Coping with anxiety and pain by adolescents with Juvenile idiopathic arthritis. Clin Exp Med Lett. 2010;51 (1):1-5. 
51. Capuron L, Schroecksnadel S, Feart C, et al. Chronic low-grade inflammation in elderly persons is associated with altered tryptophan and tyrosine metabolism: role in neuropsychiatric symptoms. Biol Psychiatry. 2011;70(2):175-182. doi:10.1016/j.biopsych.2010.12.006

52. Nerurkar L, Siebert S, McInnes IB, Cavanagh J. Rheumatoid arthritis and depression: an inflammatory perspective. Lancet Psychiat. 2019;6(2):164-173. doi:10.1016/S2215-0366(18)30255-4

53. GAH K-B, Albers E, Voskamp M, et al. Juvenile Arthritis patients suffering from chronic inflammation have increased activity of both IDO and GTP-CH1 pathways but decreased BH4 efficacy: implications for well-being, including fatigue, cognitive impairment, anxiety, and depression. Pharmaceuticals. 2019;12(1):08.

54. Duffy CM, Arsenault L, Duffy KN. Level of agreement between parents and children in rating dysfunction in juvenile rheumatoid arthritis and juvenile spondyloarthritides. J Rheumatol. 1993;20 (12):2134-2139.

55. Noll RB, Kozlowski K, Gerhardt C, Vannatta K, Taylor J, Passo M. Social, emotional, and behavioral functioning of children with juvenile rheumatoid arthritis. Arthritis Rheum. 2000;43(6):1387-1396. doi:10.1002/1529-0131(200006)43:6<1387::AID-ANR24>3.0.CO;2-C

56. Vuorimaa H, Tamm K, Honkanen V, Konttinen YT, Komulainen E, Santavirta N. Empirical classification of children with JIA: a multidimensional approach to pain and well-being. Clin Exp Rheumatol. 2008;26(5):954-961.

57. Naskar S, Victor R, Nath K. Depression in diabetes mellitus-A comprehensive systematic review of literature from an Indian perspective. Asian J Psychiatr. 2017;27:85-100. doi:10.1016/j.ajp.2017.02.018

58. Almeida OP, McCaul K, Hankey GJ, et al. Duration of diabetes and its association with depression in later life: the Health In Men Study (HIMS). Maturitas. 2016;86:3-9. doi:10.1016/j.maturitas.2016.01.003

59. David J, Cooper C, Hickey L, et al. The functional and psychological outcomes of juvenile chronic arthritis in young adulthood. $\mathrm{Br} J$ Rheumatol. 1994;33(9):876-881. doi:10.1093/rheumatology/33.9.876

60. Miller AH, Raison CL. The role of inflammation in depression: from evolutionary imperative to modern treatment target. Nat Rev Immunol. 2016;16(1):22-34. doi:10.1038/nri.2015.5

61. Raison CL, Rutherford RE, Woolwine BJ, et al. A randomized controlled trial of the tumor necrosis factor antagonist infliximab for treatmentresistant depression: the role of baseline inflammatory biomarkers. JAMA Psychiatry. 2013;70(1):31-41. doi:10.1001/2013.jamapsychiatry.4

62. Gerloni V, Pontikaki I, Gattinara M, Fantini F. Focus on adverse events of tumour necrosis factor alpha blockade in juvenile idiopathic arthritis in an open monocentric long-term prospective study of 163 patients. Ann Rheum Dis. 2008;67(8):1145-1152. doi:10.1136/ ard.2007.069484

63. Horneff G, De Bock F, Foeldvari I, et al. Safety and efficacy of combination of etanercept and methotrexate compared to treatment with etanercept only in patients with juvenile idiopathic arthritis (JIA): preliminary data from the German JIA Registry. Ann Rheum Dis. 2009;68(4):519-525. doi:10.1136/ard.2007.087593

64. Lovell DJ, Giannini EH, Reiff A, et al. Etanercept in children with polyarticular juvenile rheumatoid arthritis. Pediatric Rheumatology Collaborative Study Group. N Engl J Med. 2000;342(11):763-769. doi:10.1056/NEJM200003163421103

Open Access Rheumatology: Research and Reviews

\section{Publish your work in this journal}

Open Access Rheumatology Research and Reviews is an international, peer-reviewed, open access journal publishing original research, reports, editorials, reviews and commentaries on all aspects of clinical and experimental rheumatology in the clinic and laboratory including the following topics: Pathology, pathophysiology of rheumatological diseases; Investigation, treatment and management
65. Hider SL, Tanveer W, Brownfield A, Mattey DL, Packham JC. Depression in RA patients treated with anti-TNF is common and under-recognized in the rheumatology clinic. Rheumatology (Oxford). 2009;48(9):1152-1154. doi:10.1093/rheumatology/kep170

66. Davis AM, Graham TB, Zhu Y, McPheeters ML. Depression and medication nonadherence in childhood-onset systemic lupus erythematosus. Lupus. 2018;27(9):1532-1541. doi:10.1177/0961203318779710

67. Aasland A, Flatø B, Vandvik IH. Psychosocial outcome in juvenile chronic arthritis: A nine-year follow- up. Clin Exp Rheumatol. 1997;15(5):561-568.

68. Packham JC, Hall MA, Pimm TJ. Long-term follow-up of 246 adults with juvenile idiopathic arthritis: predictive factors for mood and pain. Rheumatology (Oxford). 2002;41(12):1444-1449. doi:10.1093/ rheumatology/41.12.1444

69. Moreira JM, Bouissou Morais Soares CM, Teixeira AL, Simoes ESAC, Kummer AM. Anxiety, depression, resilience and quality of life in children and adolescents with pre-dialysis chronic kidney disease. Pediatr Nephrol. 2015;30(12):2153-2162. doi:10.1007/ s00467-015-3159-6

70. Shelby GD, Shirkey KC, Sherman AL, et al. Functional abdominal pain in childhood and long-term vulnerability to anxiety disorders. Pediatrics. 2013;132(3):475-482. doi:10.1542/peds.2012-2191

71. Hagglund K, Schopp LM, Alberts KR, Cassidy JT, Frank RG. Predicting pain among children with juvenile rheumatoid arthritis. Arthritis Care Res (Hoboken). 1995;8(1):36-42. doi:10.1002/art.1790080109

72. Ding T, Hall A, Jacobs K, David J. Psychological functioning of children and adolescents with juvenile idiopathic arthritis is related to physical disability but not to disease status. Rheumatology (Oxford). 2008;47(5):660-664. doi:10.1093/rheumatology/ken095

73. Hysing M, Askeland KG, La Greca AM, Solberg ME, Breivik K, Sivertsen B. Bullying involvement in adolescence: implications for sleep, mental health, and academic outcomes. J Interpers Violence. 2019;886260519853409.

74. Naveed S, Waqas A, Aedma KK, Afzaal T, Majeed MH. Association of bullying experiences with depressive symptoms and psychosocial functioning among school going children and adolescents. BMC Res Notes. 2019;12(1):198. doi:10.1186/s13104-019-4236-x

75. Logan DE, Simons LE, Kaczynski KJ. School functioning in adolescents with chronic pain: the role of depressive symptoms in school impairment. J Pediatr Psychol. 2009;34(8):882-892. doi:10.1093/jpepsy/jsn143

76. Lavigne JV, Faier-Routman J. Correlates of psychological adjustment to pediatric physical disorders: a meta-analytic review and comparison with existing models. J Dev Behav Pediatr. 1993;14(2):117-123.

77. Eckshtain D, Marchette LK, Schleider J, Weisz JR. Parental depressive symptoms as a predictor of outcome in the treatment of child depression. $J$ Abnorm Child Psychol. 2018;46(4):825-837. doi:10.1007/s10802-017-0323-4

78. Goodman SH, Rouse MH, Connell AM, Broth MR, Hall CM, Heyward D. Maternal depression and child psychopathology: a meta-analytic review. Clin Child Fam Psychol Rev. 2011;14(1):127. doi:10.1007/s10567-010-0080-1

79. Goodman SH. Depression in mothers. Annu Rev Clin Psychol. 2007;3:107-135. doi:10.1146/annurev.clinpsy.3.022806.091401

of rheumatological diseases; Clinical trials and novel pharmacological approaches for the treatment of rheumatological disorders. The manuscript management system is completely online and includes a very quick and fair peer-review system, which is all easy to use. Visit http://www.dovepress.com/testimonials.php to read real quotes from published authors. 